\title{
ORDER PRESERVING VIBRATING STRINGS AND APPLICATIONS TO ELECTRODYNAMICS AND MAGNETOHYDRODYNAMICS*
}

\author{
YANN BRENIER ${ }^{\dagger}$
}

\begin{abstract}
The motion of a collection of vertical strings subject to horizontal linear vibrations in the plane can be described by a system of first order nonlinear conservations laws. This system -that we call the Chaplygin-Born-Infeld (CBI) system- is related to Magnetohydrodynamics and more specifically to its shallow water version. Then, each vibrating string can be interpreted as a magnetic line. The CBI system is also related to the Born-Infeld theory for the electromagnetic field, a nonlinear correction to the classical Maxwell's equations.

Due to the linearity of vibrations, there is a priori no mechanism to prevent the strings to cross each other, at least for sufficiently large initial impulse. These crossings generate concentration singularities in the CBI system. A numerical scheme is introduced to maintain order preserving strings beyond singularities. This order preserving scheme is shown to be convergent to a distinguished limit, which can be interpreted, through maximal monotone operator theory, as a vanishing viscosity limit of the CBI system. Finally, models of pressureless gas with sticky particles are revisited and a new formulation is provided.
\end{abstract}

Key words. conservation laws, vibrating strings, magnetohydrodynamics, maximal monotone operators

AMS subject classifications. 35L70, 74K05, 76W05, 47H06

1. Vibrating strings in the plane. Let us consider a one-parameter family of vertical vibrating strings subject to horizontal vibrations in the plane (like a harp). Each string is labelled by $a \in[0,1]$ and described at time $t$ by a curve in the plane $(x, y)$ :

$$
y \in \mathbf{R} \rightarrow(X(t, a, y), y) .
$$

For simplicity, we assume spatial periodicity in $y$, so that $y \in \mathbf{R} / \mathbf{Z}$. Each string is subject to horizontal vibrations according to the linear wave equation

$$
\partial_{t t} X=c^{2} \partial_{y y} X
$$

with propagation speed $c$. Then, we observe:

Proposition 1.1. Let $t_{0}<t_{1}$ and $c=1$. Assume that $X(t, a, y)$ is smooth and satisfies $\partial_{a} X>0$ for $(a, y) \in K=[0,1] \times \mathbf{R} / \mathbf{Z}$ and $t \in\left[t_{0}, t_{1}\right]$. Then,

$$
\begin{gathered}
h(t, X(t, a, y), y)=\frac{1}{\partial_{a} X(t, a, y)}, \\
b(t, X(t, a, y), y)=\partial_{y} X(t, a, y), \quad v(t, X(t, a, y), y)=\partial_{t} X(t, a, y),
\end{gathered}
$$

implicitly define a solution $(h, b, v)$ to the system of nonlinear first order conservation laws:

$$
\begin{gathered}
\partial_{t}(h v)+\partial_{x}\left(h v^{2}-h b^{2}\right)-\partial_{y}(h b)=0, \\
\partial_{t} h+\partial_{x}(h v)=0, \quad \partial_{t}(h b)-\partial_{y}(h v)=0,
\end{gathered}
$$

${ }^{*}$ Received October 4, 2004; accepted for publication January 11, 2005.

${ }^{\dagger}$ CNRS, LJAD, Université de Nice, France (brenier@math.unice.fr) 
for $t_{0} \leq t \leq t_{1}$, on the strip

$$
S(t)=\{X(t, 0, y) \leq x \leq X(t, 1, y)\} .
$$

The proof of this elementary observation is postponed to the first Appendix. Notice that, as long as $\partial_{a} X>0$ holds true, $(h, b, v)$ may be equivalently defined by:

$$
(h, h b, h v)(t, x, y)=\int\left(1, \partial_{y} X, \partial_{t} X\right)(t, a, y) \delta(x-X(t, a, y)) d a .
$$

2. The Chaplygin-Born-Infeld system. Before discussing the physical intepretation of system (3) -that we call the Chaplygin-Born-Infeld (CBI) system- where $h, v, b$ can be respectively interpreted as the density, velocity and magnetic fields of a magnetic fluid, let us first consider two particular types of solutions. For those solutions of (3) which do not depend on $x, h$ is a time independent field, and (3) is just a linear wave equation for $(v, b)$, written as a first order system:

$$
\partial_{t}(h v)=\partial_{y}(h b) \quad \partial_{t}(h b)=\partial_{y}(h v) .
$$

Next, for solutions that do not depend on $y, B=b h$ is a time independent field. If, in addition, $B$ is a constant, we recover a gas dynamics model:

$$
\partial_{t} h+\partial_{x}(h v)=0, \quad \partial_{t}(h v)+\partial_{x}\left(h v^{2}+p\right)=0,
$$

with density $h \geq 0$, velocity $v$, and pressure $p$ given by the unusual law $p=p(h)=$ $-B^{2} / h$. The corresponding sound speed $\sqrt{d p / d h}=|B| / h$ tends to zero as the density $h$ tends to infinity. This model is known as the Chaplygin gas and allows mass concentrations in finite time. It has been advertised as a possible model for dark energy [GKMP]. From a mathematical point of view, the Chaplygin gas equations are known to be hyperbolic, linearly degenerate ([BDLL], $[\mathrm{Se}] . .$.$) , with possible blow$ up in sup norm for the density field $h$.

Finally, by setting

$$
\begin{gathered}
\underline{x}=(x, y, z), \quad \nabla=\left(\partial_{x}, \partial_{y}, \partial_{z}\right), \\
\underline{v}(t, \underline{x})=(v(t, x, y), 0,0), \quad \underline{b}(t, \underline{x})=(b(t, x, y), 1,0),
\end{gathered}
$$

we see that (3) is just a particular case of the following system

$$
\begin{gathered}
\partial_{t} h+\nabla \cdot(h \underline{v})=0, \quad \partial_{t}(h \underline{b})+\nabla \times(h \underline{b} \times \underline{v})=0, \\
\partial_{t}(h \underline{v})+\nabla \cdot(h(\underline{v} \otimes \underline{v}-\underline{b} \otimes \underline{b}))=0 .
\end{gathered}
$$

The later system has at least two possible interpretations. First, in the $2 \mathrm{D}$ case, i.e. for solutions that do not depend on $z$, (7) just describes "shallow water" Magnetohydrodynamics (SWMHD) [Gil], without gravity terms. The shallow water MHD equations are derived from the 3D MHD equations by averaging out the vertical direction, in the case of an incompressible inviscid magnetic fluid moving inside a thin, nearly horizontal, free boundary domain (like a pancake). For that reason, each string $y \rightarrow(X(t, a, y), y) \in \mathbf{R} \times \mathbf{R} / \mathbf{Z}$ can be interpreted as an integral line of the magnetic field $\underline{b}$. Then, condition $\partial_{a} X>0$ just means that these curves do not cross each other 
and do not even touch each other. A second possible interpretation for system (7) comes from the (augmented) Born-Infeld (ABI) equations, as discussed in [Br]. The ABI system reads:

$$
\begin{aligned}
\partial_{t} h+\nabla \cdot(h \underline{v}) & =0, \\
\partial_{t}(h \underline{b})+\nabla \times(h \underline{b} \times \underline{v})+\nabla \times d & =0, \\
\partial_{t}(h \underline{d})+\nabla \times(h \underline{d} \times \underline{v})-\nabla \times b & =0, \\
\partial_{t}(h \underline{v})+\nabla \cdot(h(\underline{v} \otimes \underline{v}-\underline{b} \otimes \underline{b}-\underline{d} \otimes \underline{d})) & =\nabla\left(\frac{1}{h}\right),
\end{aligned}
$$

where $b, d, v$ are valued in $\mathbf{R}^{3}$ and $h \geq 1$. Then (7) can be derived as a high field limit of system (8), as $h>>1$ and $d<<1$. Properly speaking, the Born-Infeld (BI) equations correspond to the ABI system (8), restricted to the algebraic manifold:

$$
\tau^{2}+d^{2}+b^{2}+v^{2}=1, \quad \tau v=d \times b, \quad \tau=1 / h,
$$

which is preserved by the ABI system (at least for smooth solutions). The BI system was introduced in 1934 [BI] as a nonlinear modification of Maxwell's equations, in order to cure the problem of divergent electrostatic fields generated by point particles in classical Electrodynamics (see [Ja] for a discussion). The BI equations have a lot of interesting features, for which we refer to Boillat's lecture in [BDLL] for some mathematical aspects and [Gib] for their impact in modern high energy Physics and String Theory. Interestingly enough, it can be easily checked that, in one space dimension, the ABI system reduces to the Chaplygin gas equations previously mentioned.

3. Occurence of singularities. Through Proposition 1.1, we have a simple way to solve the Cauchy problem for the CBI system (3), at least for a substantial class of initial conditions. Let us consider, for instance, initial conditions of the following type:

$h(t=0, x, y)$ is the indicator function of the strip $\{0<x<1\}$,

$b(t=0, x, y)=1$ and $v(0, x, y)=\partial_{y} U_{0}(x, y)$ where $U_{0}$ is a smooth function such that

$$
\sup _{\left(a, y, y^{\prime}\right)}\left|\partial_{a} U_{0}(a, y)-\partial_{a} U_{0}\left(a, y^{\prime}\right)\right|<2 c .
$$

First, we set

$$
X(t=0, a, y)=a, \quad \partial_{t} X(t=0, a, y)=\partial_{y} U_{0}(a, y)
$$

Next, solving the wave equation (1), we get, by d'Alembert's formula,

$$
X(t, a, y)=a+\frac{1}{2 c}\left(U_{0}(a, y+c t)-U_{0}(a, y-c t)\right),
$$

with, here, $c=1$. Because of (9), we have $\partial_{a} X>0$ and, therefore, we obtain, through (2), a global solution to system (3). Notice that, in this case, condition (9) is a necessary and sufficient condition to get a global solution (for both positive and negative times). Otherwise, we only get a local solution which breaks down for sufficiently large values of $t$. More precisely, the strings $y \rightarrow(X(t, y, a), y)$ cross each other and $h$ blows up in sup norm. In physical terms, these singularities can be interpreted as crossing of "magnetic lines" and "density" concentrations. 
4. Beyond singularities. Let us know address the following question: how local solutions to the CBI system (3), obtained by solving the linear wave equation (1) according to Proposition 1.1, can be extended globally, beyond singularities? There is not a unique answer: it is a matter of modelling. For instance, we may be happy with the linear wave equation and keep formulae (2) to define a generalized solution $(h, b, v)$ to system (3). Such a solution cannot be a weak solution in the usual sense but, rather, a multivalued solution. A more consistent choice is to constrain the wave equation (1) in such way that the resulting solutions $X$ at least satisfy condition $\partial_{a} X \geq 0$, which means that the "magnetic lines" $y \rightarrow(X(t, y, a), y)$ are allowed to touch but not to cross each other. This is compatible with a weak formulation of (3), provided $h$ is considered as a nonnegative measure, and $h b, h v$ are real valued measures absolutely continuous with respect to $h$ ( $b$ and $v$ being their -square integrable- Radon-Nikodym derivatives with respect to $h$ ). There are many ways to constrain the wave equations, in the spirit of contact theory in Mechanics and variational inequalities in Mathematics (see [PS] as a recent example). For instance, using a variational time implicit scheme with time step $\delta t>0$, it would be natural to define $X_{n}(a, y)$ as an approximation to $X(n \delta t, a, y)$ for $n=1,2,3, \cdots$, by solving, at each step $n$, the convex minimization problem in $X(a, y)$ :

$$
\inf _{X} \sup _{\mu \geq 0} \int\left(\delta t^{-2}\left|X-2 X_{n}+X_{n-1}\right|^{2}+c^{2}\left|\partial_{y} X\right|^{2}-2 \mu \partial_{a} X\right) d a d y
$$

where $\mu=\mu(a, y) \geq 0$ is a Lagrange multiplier for condition $\partial_{a} X(a, y) \geq 0$. (Notice that, without constraint, we would recover the familiar implicit time discretization of the linear wave equation.) This would lead, at least formally as $\delta t \rightarrow 0$, to the following modification of the wave equation:

$$
\partial_{t t} X=c^{2} \partial_{y y} X-\partial_{a} \mu, \quad \partial_{a} X \geq 0, \quad \mu \geq 0, \quad \mu \partial_{a} X=0 .
$$

However, it is unclear (at least to us) that this provides a well posed formulation. So, we will consider a different approximation scheme.

5. A time discrete scheme beyond singularities. We now introduce a time discrete scheme to go beyond singularities.

Given a uniform time step $\delta t>0$, we denote by $\left(X_{\delta t, n}(a, y), U_{\delta t, n}(a, y)\right)$ the approximate solution at time $t=n \delta t$, for $n=0,1,2, \cdots$, defined in two steps as follows.

We first define $U_{\delta t, n+1}$ and $\hat{X}_{\delta t, n+1}$ by

$$
\begin{aligned}
\hat{X}_{\delta t, n+1}(a, y)=\frac{1}{2}( & \left.X_{\delta t, n}(y+c \delta t, a)+X_{\delta t, n}(y-c \delta t, a)\right) \\
& +\frac{1}{2 c}\left(U_{\delta t, n}(y+c \delta t, a)-U_{\delta t, n}(y-c \delta t, a)\right) \\
U_{\delta t, n+1}(a, y)=\frac{c}{2}( & \left.X_{\delta t, n}(y+c \delta t, a)-X_{\delta t, n}(y-c \delta t, a)\right) \\
& +\frac{1}{2}\left(U_{\delta t, n}(y+c \delta t, a)+U_{\delta t, n}(y-c \delta t, a)\right) .
\end{aligned}
$$

This amounts, so far, to solving the wave equation (1) between time $n \delta t$ and time $(n+1) \delta t$ using d'Alembert's formula. Next, we rearrange $\hat{X}_{\delta t, n+1}(a, y)$ in increasing 
order with respect to $a \in[0,1]$, for each $y \in \mathbf{R} / \mathbf{Z}$, and obtain $X_{\delta t, n+1}(a, y)$. In other words, we set

$$
X_{\delta t, n+1}=\hat{X}_{\delta t, n+1}^{*}
$$

where we denote by $Y^{*}$ the non decreasing rearrangement of a given function $Y \in$ $L^{2}([0,1])$. Let us recall that $Y^{*}$ is implicitly and uniquely defined by

$$
\partial_{a} Y^{*} \geq 0, \quad \int_{0}^{1} 1\left\{Y^{*}(a)>x\right\} d a=\int_{0}^{1} 1\{Y(a)>x\} d a, \quad \forall x \in \mathbf{R} .
$$

Observe that this time discrete scheme becomes a fully discrete scheme, if the initial data $X_{\delta t, 0}(a, y)$ and $U_{\delta t, 0}(a, y)$ are piecewise constant on a uniform cartesian grid with steps $\delta y$ and $\delta a$, provided that the time step $\delta t$ is chosen so that $c \delta t=\delta y$. Using standard finite difference notations, the resulting scheme is defined in two steps. The predictor step reads

$$
\begin{aligned}
& \tilde{X}_{n+1, k, j}=\frac{1}{2}\left(X_{n, k, j+1}+X_{n, k, j-1}\right)+\frac{1}{2 c}\left(U_{n, k, j+1}-U_{n, k, j-1}\right), \\
& U_{n+1, k, j}=\frac{1}{2}\left(U_{n, k, j+1}+U_{n, k, j-1}\right)+\frac{c}{2}\left(X_{n, k, j+1}-X_{n, k, j-1}\right),
\end{aligned}
$$

where $X_{n, k, j}$ and $U_{n, k, j}$ are respectively the (constant) values of $X_{\delta t, n}(a, y)$ and $U_{\delta t, n}(a, y)$ in the cells $|y-j \delta y|<\delta y / 2$ and $|a-k \delta a|<\delta a / 2$. The corrector step amounts to define, for each fixed $n$ and $j$, the sequence $\left(X_{n+1, k, j}\right)_{k}$ to be $\left(\tilde{X}_{n+1, k, j}\right)_{k}$ sorted in increasing order (with possible repetitions) with respect to $k$.

To illustrate this numerical scheme, we compare at a given time $T>0$ two solutions corresponding to the same initial conditions: a non order preserving solution (figure 1) on one hand and an order preserving solution, on the other hand (figure 2). We set $c=1$ and $T=0.8$. The initial conditions are:

$$
X_{0}(a, y)=a, \quad U_{0}(a, y)=4 \sin (2 \pi(a+y))
$$

and the discretization parameters are: $\delta t=\delta y=0.01$ and $\delta a=0.05$ (which corresponds to 21 "magnetic lines" and 100 mesh points to discretize the wave equation in $y$ ). The kinetic and the total energy at step $n$ are respectively defined as

$$
\begin{gathered}
K_{n}=\sum_{j, k}\left(\tilde{X}_{n, k, j}-X_{n-1, k, j}\right)^{2} \\
T_{n}=K_{n}+\sum_{j, k}\left(U_{n, k, j}-U_{n-1, k, j}\right)^{2} .
\end{gathered}
$$

It will be shown in the next section that $T_{n}$ is decreasing in $n$. On figure 3 , we show the evolution of the kinetic and the total energy. We observe a fast dissipation of the total energy until $T=0.8$ (approximately) due to a lot of string crossings. Then, there is no dissipation any longer, crossings stop and the solutions become 1-periodic in time. 


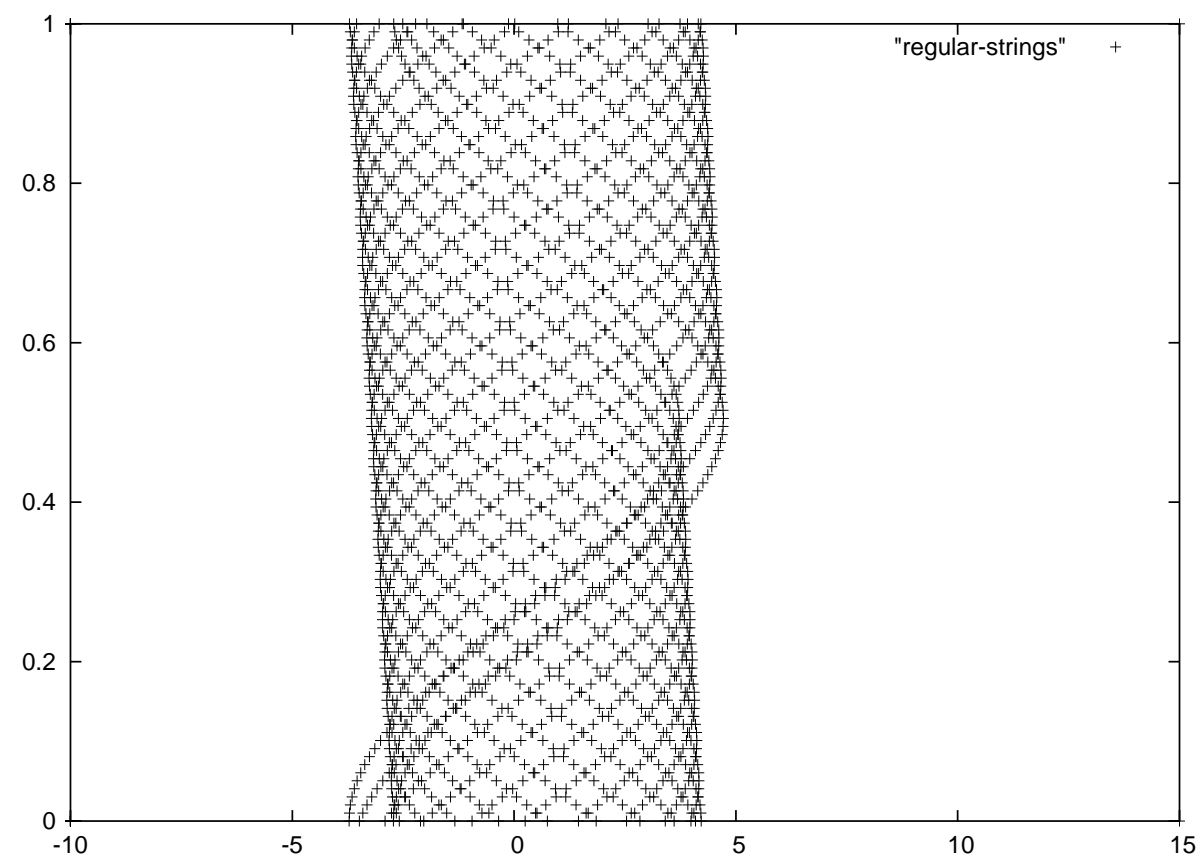

FIG. 1. Regular strings. Solution at $T=0.8$, Initial conditions: $X_{0}(a, y)=a, U_{0}(a, y)=$ $4 \sin (2 \pi(a+y))$. Horizontal axis: $-10<x<15,21$ strings. Vertical axis: $0<y<1, \delta y=0.01$, 100 grid points. Time stepping: $\delta t=\delta y=0.01$.

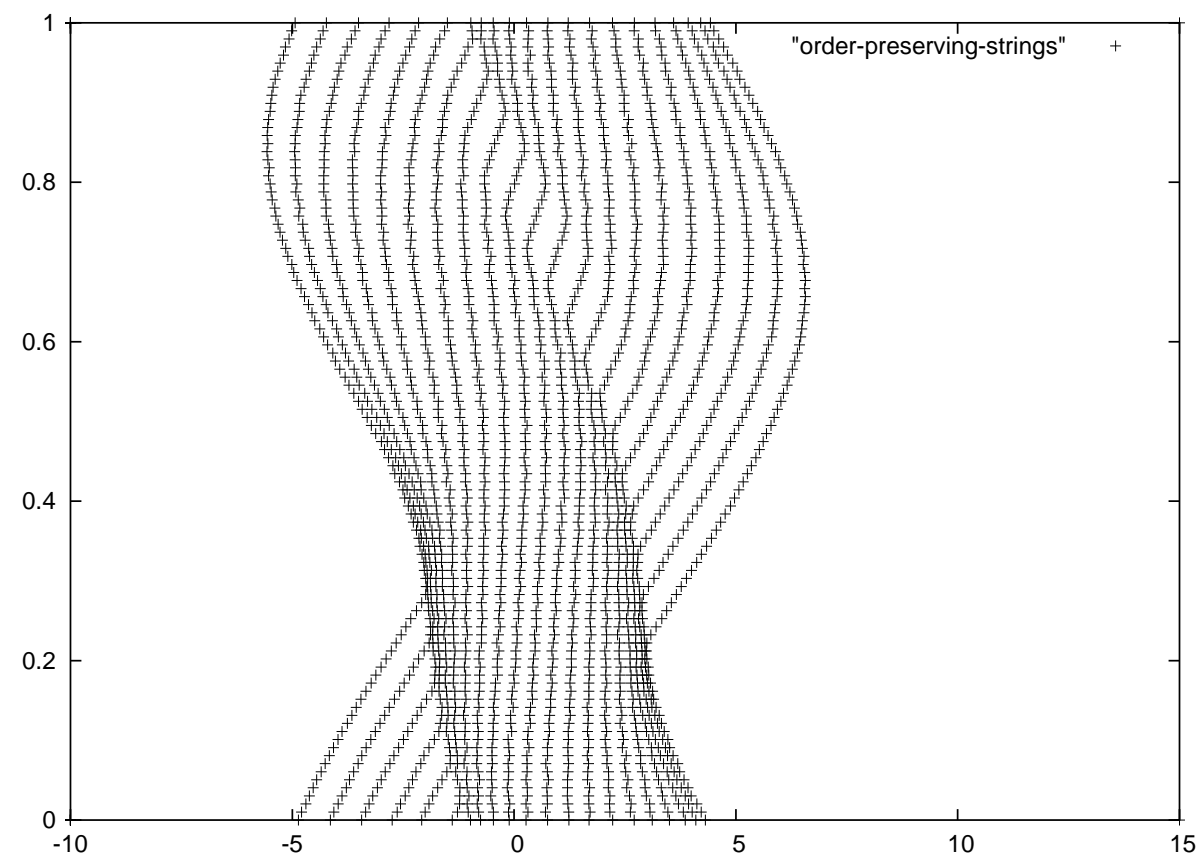

FIG. 2. Order preserving strings. Solution at $T=0.8$, Initial conditions: $X_{0}(a, y)=a$, $U_{0}(a, y)=4 \sin (2 \pi(a+y))$. Horizontal axis: $-10<x<15$, 21 strings. Vertical axis: $0<y<1$, $\delta y=0.01,100$ grid points. Time stepping: $\delta t=\delta y=0.01$. 


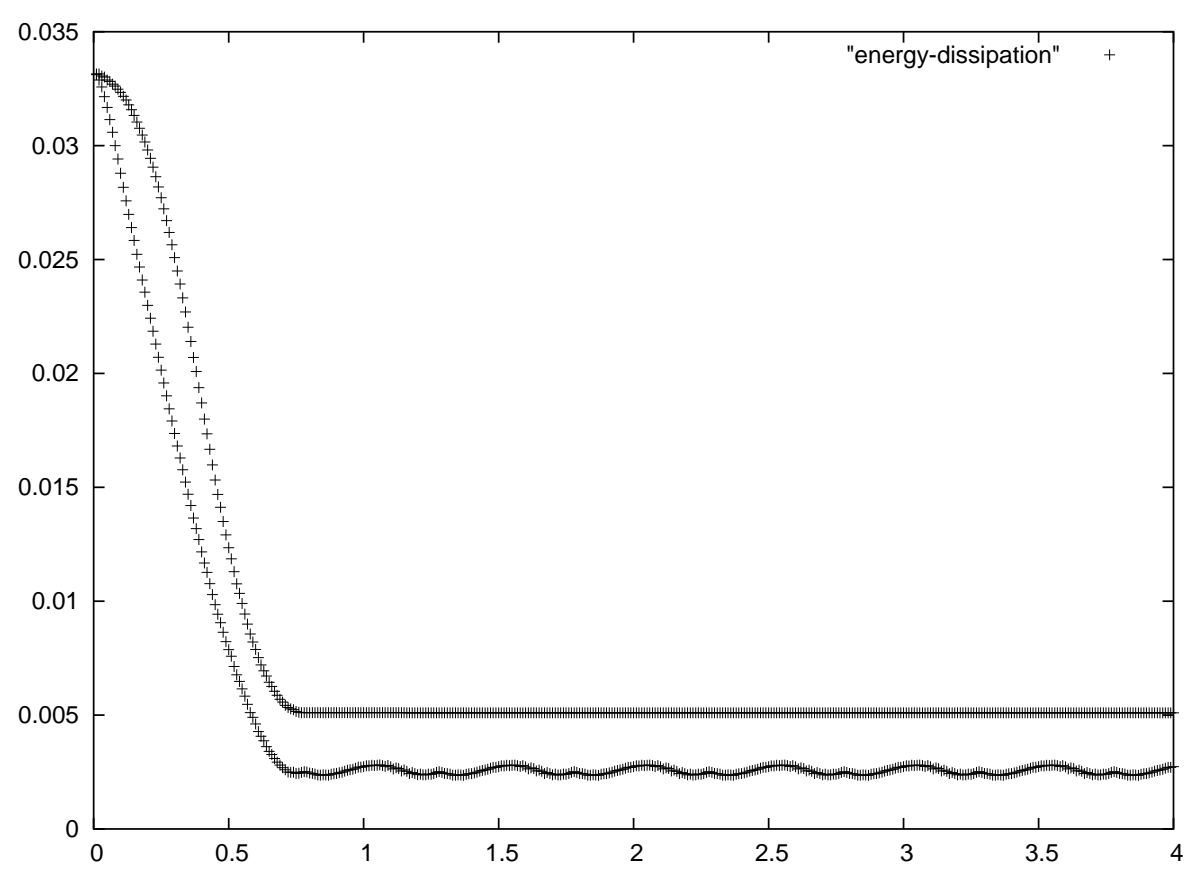

Fig. 3. Decay of the total energy. Horizontal axis: $0<t<4, \delta t=0.01$. Upper curve: Total energy, Lower curve: Kinetic energy.

6. Convergence of the time discrete scheme. In this section, we establish the convergence of the time discrete scheme to a distinguished limit. Let us first introduce:

Definition 6.1. Let $K=[0,1] \times \mathbf{R} / \mathbf{Z}$ and $\|\cdot\|$ be the norm on $L^{2}(K)$. Let $\left(X_{0}, U_{0}\right) \in L^{2}(K)^{2}$ such that $\partial_{a} X_{0} \geq 0$. We say that

$$
t \geq 0 \rightarrow(X(t), U(t))=((a, y) \in K \rightarrow(X, U)(t, a, y)) \in\left(L^{2}(K)\right)^{2}
$$

is an order preserving family of solutions to the wave equation (1) with initial values $\left(X_{0}, U_{0}\right)$ if:

i) $(X(t), U(t))$ depends continuously on $t$ with respect to the weak topology of $L^{2}(K)$, and $\partial_{a} X \geq 0$ holds true,

ii) $X(0)=X_{0}, U(0)=U_{0}$,

iii) for all $T \geq 0$ and all pair of smooth functions $(Y, V)(t, x, a)$ such that $\partial_{a} Y \geq 0$, we have:

$$
\begin{aligned}
& \|X(T)-Y(T)\|^{2}+c^{-2}\|U(T)-V(T)\|^{2} \leq\left\|X_{0}-Y(0)\right\|^{2}+c^{-2}\left\|U_{0}-V(0)\right\|^{2} \\
& \quad+2 \int_{0}^{T} d t \int_{K} d y d a\left(U \partial_{y} Y+(Y-X) \partial_{t} Y+X \partial_{y} V+c^{-2}(V-U) \partial_{t} V\right)
\end{aligned}
$$

If, in addition,

$$
\sup _{t}\left\|\partial_{y} X(t)\right\|^{2}+c^{-2}\left\|\partial_{y} U(t)\right\|^{2}<+\infty
$$

then we say that $(X, U)$ is a strong order preserving $(S O P)$ solution. 
Then, we can prove our main result:

Theorem 6.2. Let $K=[0,1] \times \mathbf{R} / \mathbf{Z}$ and $\left(X_{0}, U_{0}\right) \in L^{2}(K)$ such that:

$$
\partial_{a} X_{0} \geq 0, \quad \partial_{y} X_{0} \in L^{2}(K), \quad \partial_{y} U_{0} \in L^{2}(K) .
$$

Then there is a unique strong order preserving solution $t \geq 0 \rightarrow(X, U)(t)$ to the wave equation (in the sense of definition 6.1) such that:

$$
(X, U)(0)=\left(X_{0}, U_{0}\right) .
$$

This solution is obtained as the limit of the time discrete scheme, as the time step $h$ goes to zero. In addition:

$$
\left\|\partial_{y} X(t)\right\|^{2}+c^{-2}\left\|\partial_{y} U(t)\right\|^{2} \leq\left\|\partial_{y} X_{0}\right\|^{2}+c^{-2}\left\|\partial_{y} U_{0}\right\|^{2} .
$$

Moreover, for all pair $(X, U),\left(X^{\prime}, U^{\prime}\right)$ of such solutions with initial values respectively given by $\left(X_{0}, U_{0}\right),\left(X_{0}^{\prime}, U_{0}^{\prime}\right)$, we have, for all $T \geq 0$,

$$
\left\|X(T)-X^{\prime}(T)\right\|^{2}+c^{-2}\left\|U(T)-U^{\prime}(T)\right\|^{2} \leq\left\|X_{0}-X_{0}^{\prime}\right\|^{2}+c^{-2}\left\|U_{0}-U_{0}^{\prime}\right\|^{2} .
$$

Remark. Finite collections of strings. So far, we have considered the case of a continuum of strings, with a continuous label $a \in[0,1]$. It is remarkable that the continuous formulation (15), in Definition 6.1, can be kept unchanged in the case of a finite collection of strings, with labels $\alpha=1, \cdots, N$. Only the monotonicity constraint has to be understood as:

$$
X(t, y, \alpha) \geq X(t, y, \alpha-1), \quad Y(t, y, \alpha) \geq Y(t, y, \alpha-1), \quad \alpha=2, \cdot \cdot, N, \quad y \in \mathbf{R} / \mathbf{Z} .
$$

To illustrate this case, we show at three different times $(T=0.1, T=0.6, T=1.1)$ the location of three interacting strings, with initial data

$$
X_{0}(a, y)=a, \quad U_{0}(a, y)=4 \sin (2 \pi(a+y)), \quad a \in\{0,0.5,1\} .
$$

We see at time $T=0.1$ that the strings are partly stuck. At this stage, the total energy is being dissipated. At later time $T=0.6$ and $T=1.1$, the strings just touch each other at a single point without sticking and the total energy is no longer dissipated.

\section{Proof of the main result.}

Compactness of the time discrete scheme. The rearrangement operator is known to be non expansive in all $L^{p}$ spaces. In particular

$$
\int_{0}^{1}\left|Y^{*}(a)-Z^{*}(a)\right|^{2} d a \leq \int_{0}^{1}|Y(a)-Z(a)|^{2} d a
$$

holds true for all pairs $(Y, Z) \in L^{2}([0,1])^{2}$. In addition, we have

$$
\int_{0}^{1}\left|Y^{*}(a)\right|^{2} d a=\int_{0}^{1}|Y(a)|^{2} d a .
$$

Since the wave operator is isometric, we easily deduce that the time discrete scheme is bounded and non expansive in $L^{2}$. Indeed, given a solution $\left(X_{\delta t, n}, U_{\delta t, n} ; n \in \mathbf{N}\right)$ of the time discrete scheme, we get

$$
\left\|X_{\delta t, n+1}\right\|^{2}+c^{-2}|| U_{\delta t, n+1}\left\|^{2}=\right\| \hat{X}_{\delta t, n+1}\left\|^{2}+c^{-2}\right\| U_{\delta t, n+1}\left\|^{2}=\right\| X_{\delta t, n}\left\|^{2}+c^{-2}\right\| U_{\delta t, n} \|^{2} .
$$




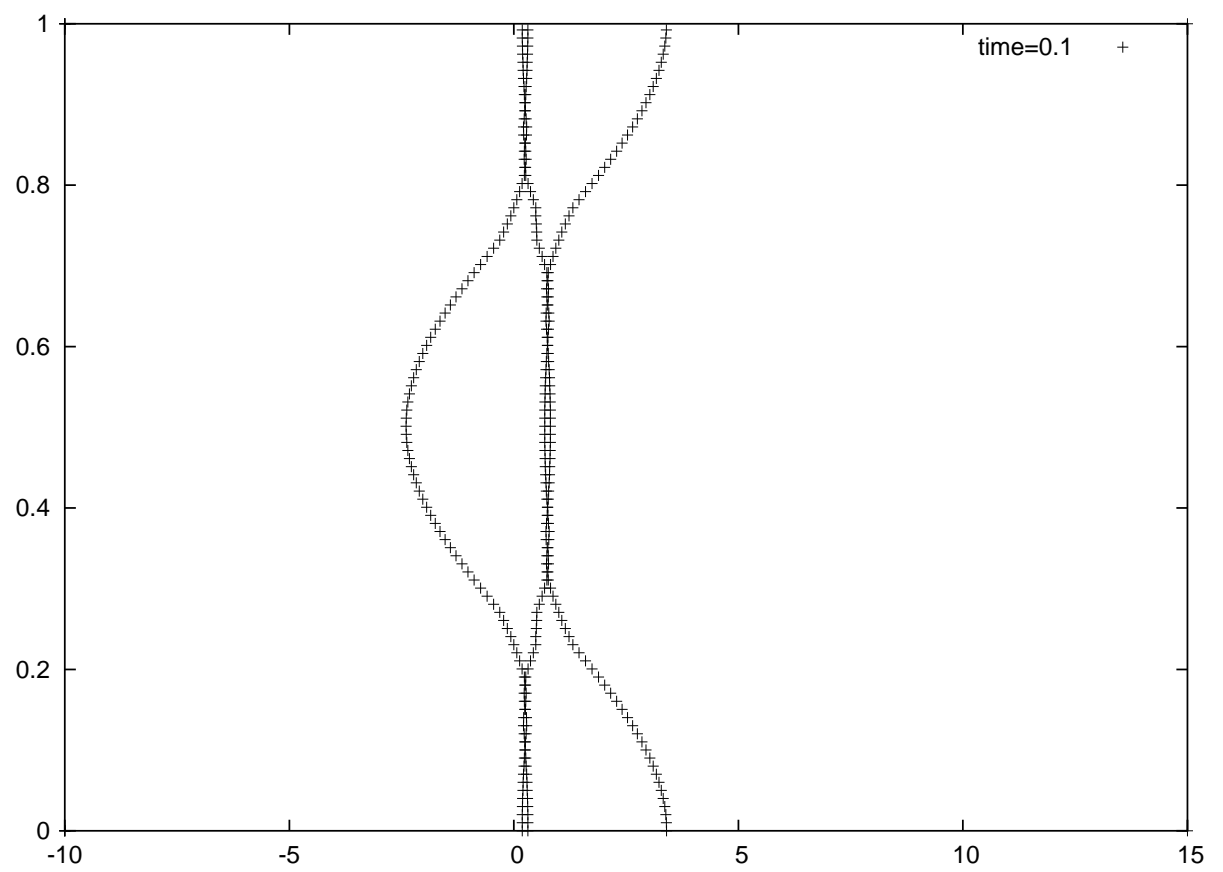

FIG. 4. Three strings. Three interacting strings : solution at $T=0.1$, Initial conditions: $X_{0}(a, y)=a, U_{0}(a, y)=4 \sin (2 \pi(a+y))$. Horizontal axis: $-10<x<15$, 3 strings. Vertical axis: $0<y<1, \delta y=0.0025,400$ grid points. Time stepping: $\delta t=\delta y=0.0025$.

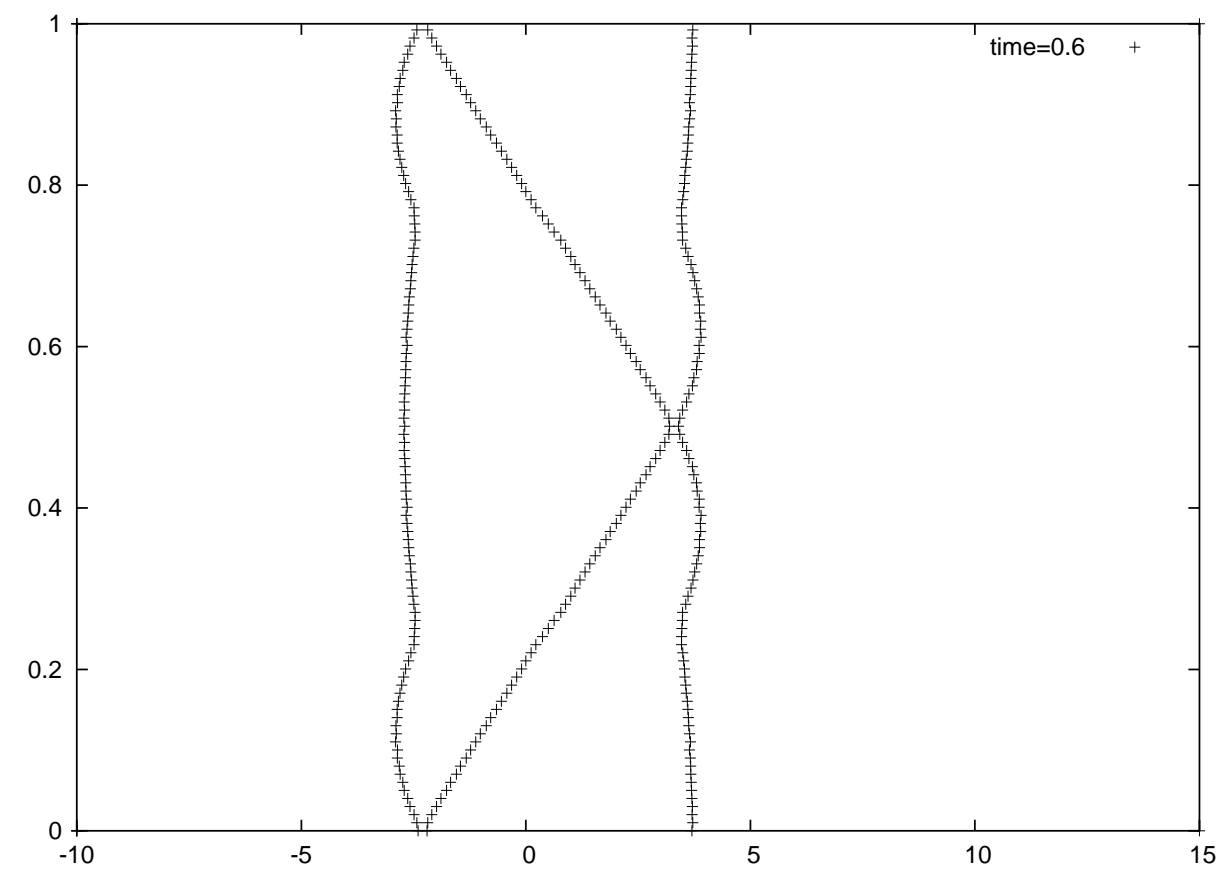

FIG. 5. Three strings. Three interacting strings : solution at $T=0.6$, Initial conditions: $X_{0}(a, y)=a, U_{0}(a, y)=4 \sin (2 \pi(a+y))$. Horizontal axis: $-10<x<15$, 3 strings. Vertical axis: $0<y<1, \delta y=0.0025,400$ grid points. Time stepping: $\delta t=\delta y=0.0025$. 


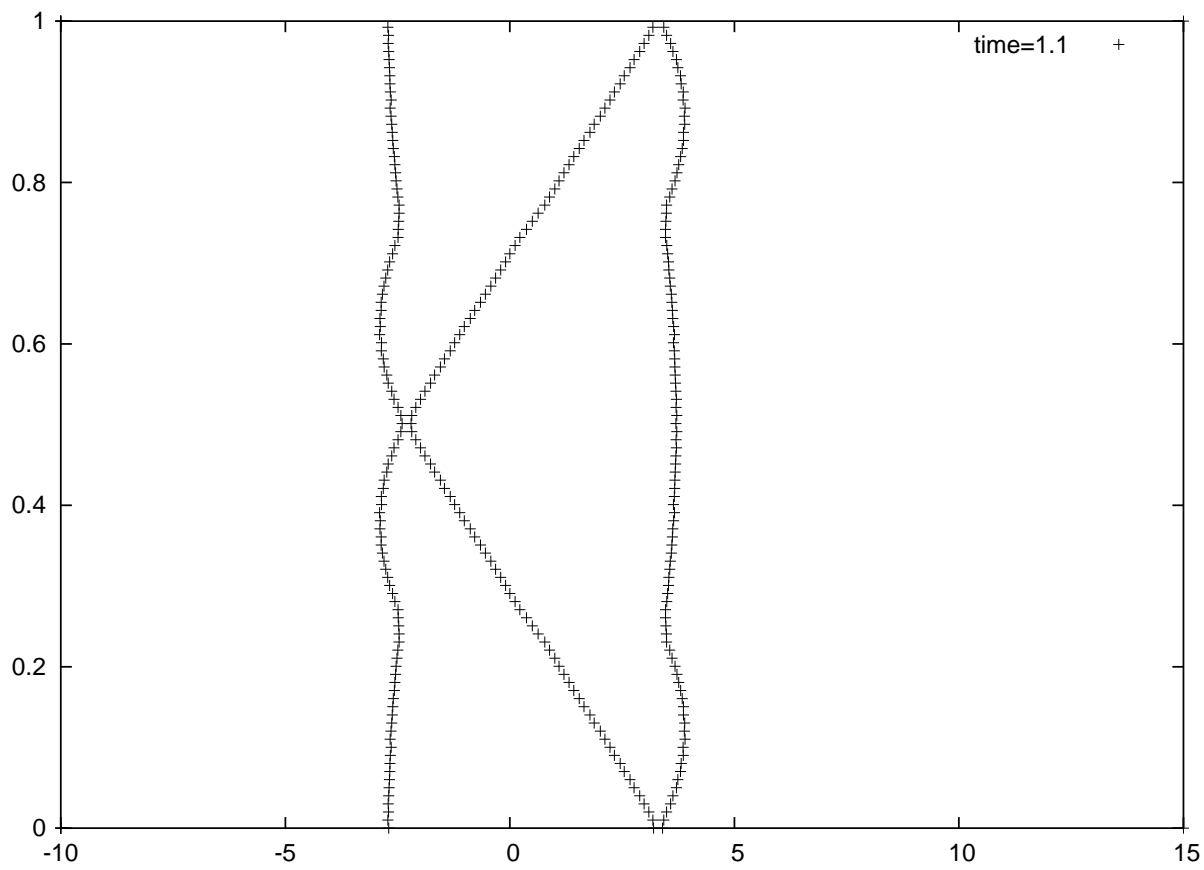

FIG. 6. Three strings. Three interacting strings : solution at $T=1.1$, Initial conditions: $X_{0}(a, y)=a, U_{0}(a, y)=4 \sin (2 \pi(a+y))$. Horizontal axis: $-10<x<15$, 3 strings. Vertical axis: $0<y<1, \delta y=0.0025,400$ grid points. Time stepping: $\delta t=\delta y=0.0025$.

Thus, we have

$$
\left\|X_{\delta t, n}\right\|^{2}+c^{-2}\left\|U_{\delta t, n}\right\|^{2}=\left\|X_{\delta t, 0}\right\|^{2}+c^{-2}\left\|U_{\delta t, 0}\right\|^{2}
$$

for all $n=0,1,2, \ldots$, which already shows that the scheme is bounded in $L^{2}$. Futhermore, if $\left(Y_{\delta t, n}, V_{\delta t, n} ; n \in \mathbf{N}\right)$ is another solution of the scheme, we get:

$$
\begin{aligned}
& \left\|X_{\delta t, n+1}-Y_{\delta t, n+1}\right\|^{2}+c^{-2}\left\|U_{\delta t, n+1}-V_{\delta t, n+1}\right\|^{2} \\
& \leq\left\|\hat{X}_{\delta t, n+1}-\hat{Y}_{\delta t, n+1}\right\|^{2}+c^{-2}\left\|U_{\delta t, n+1}-V_{\delta t, n+1}\right\|^{2} \\
& =\left\|X_{\delta t, n}-Y_{\delta t, n}\right\|^{2}+c^{-2}\left\|U_{\delta t, n}-V_{\delta t, n}\right\|^{2} \leq \cdots \\
& =\left\|X_{\delta t, 0}-Y_{\delta t, 0}\right\|^{2}+c^{-2}\left\|U_{\delta t, 0}-V_{\delta t, 0}\right\|^{2}
\end{aligned}
$$

for all $n=0,1,2, \ldots$. This shows that the scheme is non expansive in $L^{2}$. Next, by setting

$$
Y_{0}=X_{1}, \quad V_{0}=U_{1},
$$

we deduce

$$
\left\|X_{\delta t, n+1}-X_{\delta t, n}\right\|^{2}+c^{-2}\left\|U_{\delta t, n+1}-U_{\delta t, n}\right\|^{2} \leq\left\|X_{\delta t, 1}-X_{\delta t, 0}\right\|^{2}+c^{-2}\left\|U_{\delta t, 1}-U_{\delta t, 0}\right\|^{2} .
$$

Let us now initialize the time discrete scheme by setting

$$
X_{\delta t, 0}=X_{0}, \quad U_{\delta t, 0}=U_{0},
$$


where $\left(X_{0}, U_{0}\right) \in L^{2}(K)^{2}$ is chosen so that $\partial_{a} X_{0} \geq 0$ and $\partial_{y}\left(X_{0}, U_{0}\right) \in L^{2}(K)^{2}$. We see that

$$
\begin{aligned}
& \left\|X_{\delta t, 1}-X_{\delta t, 0}\right\|^{2}+c^{-2}|| U_{\delta t, 1}-U_{\delta t, 0} \|^{2} \\
& \leq \kappa_{0} \int d y d a\left(\left|X_{0}(y+c \delta t, a)-X_{0}(a, y)\right|^{2}+c^{-2}\left|U_{0}(y+c \delta t, a)-U_{0}(a, y)\right|^{2}\right)
\end{aligned}
$$

where $\kappa_{0}$ is a purely numerical constant. Thus, we have obtained

$$
\left\|X_{\delta t, n+1}-X_{\delta t, n}\right\|^{2}+c^{-2}\left\|U_{\delta t, n+1}-U_{\delta t, n}\right\|^{2} \leq \kappa_{0} \delta t^{2}\left(c^{2}\left\|\partial_{y} X_{0}\right\|^{2}+\left\|\partial_{y} U_{0}\right\|^{2}\right) .
$$

Let us now define, for all values of $t \geq 0,\left(X_{\delta} t, U_{\delta} t\right)(t, a, y)$ to be the linear interpolant in $t$ of $\left(X_{\delta t, n}, U_{\delta t, n}\right)$ at $t=n \delta t$. Then, Properties (20) and (23) are sufficient to ensure that

$$
\sup _{t \geq 0}\left\|X_{\delta} t(t)\right\|+\left\|U_{\delta} t(t)\right\|+\left\|\partial_{t} X_{\delta} t(t)\right\|+\left\|\partial_{t} U_{\delta} t(t)\right\|<+\infty
$$

which shows that $\left(X_{\delta} t, U_{\delta} t\right)$, is a (relatively) compact family in the space of all functions of $t \geq 0$ valued in $L^{2}(K)$ that are continuous with respect to the weak topology of $L^{2}(K)$. Thus, there is a sequence of time steps $\delta t \rightarrow 0$ such that $\left(X_{\delta} t, U_{\delta} t\right)$ converges in this space to some limit $(X, U)$. This limit clearly satisfies $\partial_{a} X \geq 0$. In addition, due to the obvious translation invariance of the time discrete scheme with respect to the $y$ variable, we get, for all $z \in \mathbf{R}$,

$$
\begin{aligned}
& \int d y d a\left(\left|X_{\delta t, n}(y+z, a)-X_{\delta t, n}(a, y)\right|^{2}+c^{-2}\left|U_{\delta t, n}(y+c \delta t, a)-U_{\delta t, n}(a, y)\right|^{2}\right) \\
& \leq \int d y d a\left(\left|X_{0}(y+z, a)-X_{0}(a, y)\right|^{2}+c^{-2}\left|U_{0}(y+c \delta t, a)-U_{0}(a, y)\right|^{2}\right) \\
& \leq|z|^{2}\left(\left\|\partial_{y} X_{0}\right\|^{2}+c^{-2}\left\|\partial_{y} U_{0}\right\|^{2}\right) .
\end{aligned}
$$

It follows that, for all $t \geq 0, \partial_{y}(X, U)(t)$ belongs to $L^{2}(K)^{2}$ and (18) immediately follows.

Convergence. It remains to prove that $(X, U)$ is an order preserving solutions in the sense of Definition 6.1. Let us consider a smooth pair $(Y, V)(t, a, y)$ such that $\partial_{a} Y \geq 0$. Since the rearrangement operator is non expansive, we first get

$$
\begin{aligned}
& \left\|X_{\delta t, n+1}-Y\left(t_{n+1}\right)\right\|^{2}+c^{-2}\left\|U_{\delta t, n+1}-V\left(t_{n+1}\right)\right\|^{2} \\
& \leq|| \hat{X}_{\delta t, n+1}-Y\left(t_{n+1}\right)\left\|^{2}+c^{-2}\right\| U_{\delta t, n+1}-V\left(t_{n+1}\right) \|^{2} \\
& =\int d y d a\left(\left|\frac{X(y+c \delta t)+X(y-c \delta t)+U(y+c \delta t)-U(y-c \delta t)}{2}-Y\left(t_{n+1}, y\right)\right|^{2}\right. \\
& \left.\quad+c^{-2}\left|\frac{U(y+c \delta t)+U(y-c \delta t)+c^{2}(X(y+c \delta t)-X(y-c \delta t)}{2}-V\left(t_{n+1}, y\right)\right|^{2}\right),
\end{aligned}
$$

where, for notational simplicity, some subscripts $\delta t, n$, some dependence with respect to $a$ have been dropped and $t_{k}$ stands for $k \delta t$. After few manipulations and Taylor expansions for $Y$ and $V$, we get

$$
\begin{aligned}
& \left\|X_{\delta t, n+1}-Y\left(t_{n+1}\right)\right\|^{2}+c^{-2}\left\|U_{\delta t, n+1}-V\left(t_{n+1}\right)\right\|^{2} \\
\leq & \left\|X_{\delta t, n}-Y_{t_{n}}\right\|^{2}+c^{-2}\left\|U_{\delta t, n}-V_{t_{n}}\right\|^{2} \\
& +2 \delta t \int d y d a\left(U_{\delta t, n} \partial_{y} Y(n \delta t)+\left(Y(n \delta t)-X_{\delta t, n}\right) \partial_{t} Y(n \delta t)+X_{\delta t, n} \partial_{y} V(n \delta t)\right. \\
& \left.+c^{-2}\left(V(n \delta t)-U_{\delta t, n}\right) \partial_{t} V(n \delta t)\right)+\kappa \delta t^{2},
\end{aligned}
$$


where $\kappa$ depends only on $(Y, V)$ and initial conditions $\left(X_{0}, U_{0}\right)$. Then, after adding up the last inequality for $n$ ranging from 0 to $N$, and letting $\delta t \rightarrow 0, N \delta t \rightarrow T$, where $T>0$ is fixed, we finally obtain (15), as desired.

At this point, we have shown the existence of SOP solutions obtained as limits of the numerical scheme, as the time step goes to zero.

Uniqueness and stability of SOP solutions. Since the existence of SOP solutions has been already established through the convergence analysis of the approximation scheme, it is enough to prove the stability property (19) with respect to initial conditions. This is a straightforward consequence of (15) because of bound (18). Indeed, by substituting $\left(X^{\prime}, U^{\prime}\right)$ for $(Y, V)$ in formulation (15), we get (after suitable regularizations)

$$
\begin{aligned}
& \left\|X(T)-X^{\prime}(T)\right\|^{2}+c^{-2}\left\|U(T)-U^{\prime}(T)\right\|^{2}-\left\|X_{0}-X_{0}^{\prime}\right\|^{2}-c^{-2}\left\|U_{0}-U_{0}^{\prime}\right\|^{2} \\
& \leq 2 \int_{0}^{T} d t \int_{K} d y d a\left(U \partial_{y} X^{\prime}+\left(X^{\prime}-X\right) \partial_{t} X^{\prime}+X \partial_{y} U^{\prime}+c^{-2}\left(U^{\prime}-U\right) \partial_{t} U^{\prime}\right)
\end{aligned}
$$

Symmetrizing the right hand side in $(X, U),\left(X^{\prime}, U^{\prime}\right)$ leads to:

$$
\begin{aligned}
& \left\|X(T)-X^{\prime}(T)\right\|^{2}+c^{-2}\left\|U(T)-U^{\prime}(T)\right\|^{2}-\left\|X_{0}-X_{0}^{\prime}\right\|^{2}-c^{-2}\left\|U_{0}-U_{0}^{\prime}\right\|^{2} \\
& \leq \int_{0}^{T} d t \int_{K} d y d a\left(\partial_{y}\left(U X^{\prime}+X U^{\prime}\right)+\left(X^{\prime}-X\right) \partial_{t}\left(X^{\prime}-X\right)+c^{-2}\left(U^{\prime}-U\right) \partial_{t}\left(U^{\prime}-U\right)\right) \\
& =\int_{0}^{T} d t \int_{K} d y d a\left(\left(X^{\prime}-X\right) \partial_{t}\left(X^{\prime}-X\right)+c^{-2}\left(U^{\prime}-U\right) \partial_{t}\left(U^{\prime}-U\right)\right)
\end{aligned}
$$

(since $X, U, X^{\prime}, U^{\prime}$ are periodic in $y$ )

$$
=\frac{1}{2}\left(\left\|X(T)-X^{\prime}(T)\right\|^{2}+c^{-2}\left\|U(T)-U^{\prime}(T)\right\|^{2}-\left\|X_{0}-X_{0}^{\prime}\right\|^{2}-c^{-2}\left\|U_{0}-U_{0}^{\prime}\right\|^{2}\right) .
$$

The proof of Theorem 6.2 is now complete.

Existence of OP solutions. Notice that, because of the stability property (19), it is easy to show the existence of order preserving solutions for all initial conditions $\left(X_{0}, U_{0}\right)$ in $L^{2}(K)^{2}$, provided that $\partial_{a} X_{0} \geq 0$, through a standard approximation argument. However, the uniqueness of those solutions is not clear.

Let us point out that it is not clear at all that, given an order preserving solution $(X, U)$, the corresponding field $(h, h b, h v)$, defined as measures by (4), are solutions to the original CBI system (3) in the sense of distributions. We leave this as a challenging question!

\section{Interpretations of order preserving solutions.}

Formulation with a Lagrange multiplier. Loosely speaking, formulation (15) means

$$
\partial_{t} X=\partial_{y} U-\partial_{a} \mu, \quad \partial_{t} U=c^{2} \partial_{y} X, \quad \partial_{a} X \geq 0,
$$

for some nonnegative measure $\mu(t, a, y)$, which plays the role of a Lagrange multiplier for constraint $\partial_{a} X \geq 0$. Equivalently, (24) reads:

$$
\partial_{t t} X=c^{2} \partial_{y y} X-\partial_{t} \partial_{a} \mu, \quad \partial_{a} X \geq 0, \quad \mu \geq 0,
$$

which, indeed, differs from (10) we considered earlier. Let us briefly check that Definition 6.1 is indeed consistent with formulation (24). Let us consider a SOP solution 
$(X, U)$. Thanks to (16), by using a standard mollification argument, we may substitute in (15), for $(Y, V)$, respectively $(X, U+\epsilon \omega)$ and $(X+\epsilon \xi, U)$, where $\omega$ and $\xi$ are smooth compactly supported functions of $(t, a, y) \in] 0, T[\times] 0,1\left[\times \mathbf{R} / \mathbf{Z}\right.$, with $\partial_{a} \xi \geq 0$ and $\epsilon \geq 0$. The first choice $(Y, V)=(X, U+\epsilon \omega)$ leads to

$$
\begin{aligned}
& 0 \leq 2 \int_{0}^{T} d t \int_{K}\left(U \partial_{y} X+X \partial_{y} U+\epsilon X \partial_{y} \omega+\epsilon c^{-2} \omega \partial_{t} U+\epsilon^{2} c^{-2} \omega \partial_{t} \omega\right) d y d a \\
& =2 \epsilon \int_{0}^{T} d t \int_{K}\left(X \partial_{y} \omega+c^{-2} \omega \partial_{t} U\right) d y d a+O\left(\epsilon^{2}\right)
\end{aligned}
$$

(using that $(X, U)$ is periodic in $y$ ). It follows that

$$
\partial_{t} U=c^{2} \partial_{y} X
$$

holds true in the distributional sense. Next, using $(Y, V)=(X+\epsilon \xi, U)$, we deduce from (15):

$$
\begin{aligned}
& 0 \leq 2 \int_{0}^{T} d t \int_{K}\left(U \partial_{y} X+\epsilon U \partial_{y} \xi+\epsilon \xi \partial_{t} X+\epsilon^{2} \xi \partial_{t} \xi+X \partial_{y} U\right) d y d a \\
& =2 \epsilon \int_{0}^{T} d t \int_{K}\left(U \partial_{y} \xi+\xi \partial_{t} X\right) d y d a+O\left(\epsilon^{2}\right) \\
& =2 \epsilon \int_{0}^{T} d t \int_{K}\left(-\partial_{y} U+\partial_{t} X\right) \xi d y d a+O\left(\epsilon^{2}\right)
\end{aligned}
$$

Since $\xi$ is arbitrarily chosen, with $\partial_{a} \xi \geq 0$, we deduce that there is a nonnegative measure $\mu(t, a, y)$ such that

$$
-\partial_{y} U+\partial_{t} X=-\partial_{a} \mu .
$$

Thus, formulation (24) is recovered, Notice that $\mu \partial_{a} \xi=0$ holds true when when

$$
\Omega=\{(t, a, y) \in] 0, T[\times] 0,1\left[\times \mathbf{R} / \mathbf{Z}, \quad \partial_{a} X>0\right\} .
$$

is an open set. Indeed, in such a case, we can relax condition $\partial_{a} \xi \geq 0$ in $\Omega$ and deduce that $\mu=0$ in $\Omega$.

A formulation using maximal monotone operators. More accurately, formulation (15) may be reset in the framework of maximal monotone operators [Bz] in the following way.

We use the Hilbert space $H=L^{2}(K)^{2}$ and define

$$
D=\left\{(X, U) \in H, \quad\left(\partial_{y} X, \partial_{y} U\right) \in H, \quad \partial_{a} X \geq 0\right\} .
$$

The operator $A$ itself is defined, with domain $D$, as $A=W+\partial \Phi_{0}$, where $W$ is the (skew-symmetric) linear wave operator:

$$
W(X, U)=-\left(\partial_{y} U, \partial_{y} X\right)
$$

and $\partial \Phi_{0}$ is the subdifferential of the lsc convex function $\Phi_{0}$ defined on $H$ with value 0 when $\partial_{a} X \geq 0$ and $+\infty$ otherwise. Notice that $A$ has a "gradient part", namely 
$\partial \Phi_{0}$, and a skew-symmetric part, namely $W$. For the $L^{2}$ inner product $((\cdot, \cdot)), A$ is indeed monotone, since, for all pairs $(X, U),\left(X^{\prime}, U^{\prime}\right)$,

$$
\begin{aligned}
& \left(\left(A(X, U)-A\left(X^{\prime}, U^{\prime}\right),(X, U)-\left(X^{\prime}, U^{\prime}\right)\right)\right) \\
& =\left(\left(\partial \Phi_{0}(X, U)-\partial \Phi_{0}\left(X^{\prime}, U^{\prime}\right),(X, U)-\left(X^{\prime}, U^{\prime}\right)\right)\right) \geq 0
\end{aligned}
$$

(using that $W$ is skew symmetric and $\Phi_{0}$ convex). Then, (24) can be interpreted just as:

$$
0 \in \frac{d}{d t}(X, U)+A(X, U),
$$

in the sense of maximal monotone operator theory [Bz]. Using general results of this theory, we infer that solutions of equation (28) can be approximated by those of perturbed equations such as

$$
0 \in \frac{d}{d t}(X, U)+A_{\varepsilon}(X, U), \quad A_{\varepsilon}=W+\varepsilon \partial \Phi
$$

as $\varepsilon>0$ goes to zero, for all lsc convex function $\Phi(X, U)$, provided $\Phi(X, U)=+\infty$ if and only if $\partial_{a} X \geq 0$ is violated. Typical examples are:

$$
\Phi(X, U)=\int_{K} \phi\left(\partial_{a} X(a, y)\right) d a d y
$$

for all lsc convex function $\phi$ on $\mathbf{R}$ such that $\phi(\tau)=+\infty$ if and only if $\tau<0$. A typical example is:

$$
\phi(\tau)=\tau \log \tau,
$$

for $\tau>0$, with $\phi(0)=0$ and $\phi(\tau)=+\infty$ if $\tau<0$. Other examples are:

$$
\phi(\tau)=\tau^{\gamma},
$$

with $\gamma \geq 1$, for $\tau \geq 0$, with $\phi(\tau)=+\infty$ if $\tau<0$.

Order preserving and vanishing viscosity solutions. The perturbed model $(29,27,30)$ can be written in PDE style as:

$$
\partial_{t} X=\partial_{y} U-\varepsilon \partial_{a}\left(\phi^{\prime}\left(\partial_{a} X\right)\right), \quad \partial_{t} U=\partial_{y} X .
$$

As proven in the second Appendix, for each sufficiently smooth solution $(X, U)$ satisfying $\partial_{a} X>0$, the corresponding Eulerian fields $(h, b, v)$ defined by (4) (or equivalently by (2)) are solutions to a viscous perturbation of the CBI system, namely:

$$
\begin{gathered}
\partial_{t}(h v)+\partial_{x}\left(h v^{2}-h b^{2}\right)-\partial_{y}(h b)=\varepsilon \partial_{x}\left(\mu(h) \partial_{x} v\right), \\
\partial_{t} h+\partial_{x}(h v)=0, \quad \partial_{t}(h b)-\partial_{y}(h v)=0,
\end{gathered}
$$

where the viscosity coefficient $\mu$ can be related to $\phi$ through

$$
\mu(h)=\tau \phi^{\prime \prime}(\tau), \quad \tau=\frac{1}{h} .
$$

(Notice that $\phi(\tau)=\tau \log \tau$ corresponds to a constant viscosity $\mu$.)

So, we conclude that the order preserving solutions that we have introduced to solve the CBI system can be interpreted -at least formally- as vanishing viscosity limits of the viscous model (32). 
9. Sticky particles revisited. The limit, as $c$ goes to 0 , of our numerical scheme is well defined and reduced to:

$$
\begin{gathered}
U_{\delta t, n}(a, y)=U_{0}(a, y) \\
\hat{X}_{\delta t, n+1}(a, y)=X_{\delta t, n}(a, y)+\delta t \partial_{y} U_{0}(a, y) \\
X_{\delta t, n+1}(\cdot, y)=\left(\hat{X}_{\delta t, n+1}\right)^{*}(\cdot, y), \quad \forall y \in \mathbf{R} / \mathbf{Z} .
\end{gathered}
$$

Notice that, in this scheme, $y$ acts just as a parameter, that we can disregard. Then, the scheme just reads:

$$
\begin{gathered}
\hat{X}_{\delta t, n+1}(a)=X_{\delta t, n}(a)+\delta t v_{0}(a), \\
X_{\delta t, n+1}=\hat{X}_{\delta t, n+1}^{*} .
\end{gathered}
$$

The resulting scheme is nothing but an expression of the "transport-collapse" method for scalar conservation laws [Br3], [Br2], in the particular case of monotonic initial data in one space dimension. More precisely, let $u(t, x)$ be the unique solution, à la Kruzhkov [Se] to the scalar conservation law

$$
\partial_{t} u+\partial_{x} F(u)=0
$$

with initial condition

$$
u(0, x)=\int_{0}^{1} H\left(x-X_{0}(a)\right) d a,
$$

where $H$ denotes the Heaviside function, and "flux function" $F$ defined by:

$$
F(a)=\int_{0}^{a} v_{0}\left(a^{\prime}\right) d a^{\prime}
$$

Then, it is established in [Br3] that, for each fixed $T \geq 0$,

$$
\int_{-\infty}^{+\infty} d x \int_{0}^{1}\left|H\left(x-X_{\delta t, n}(a)\right)-H(u(T, x)-a)\right| d a \rightarrow 0, \quad \delta t=T / n \rightarrow 0 .
$$

Scalar conservation laws with monotonic initial conditions have been shown in [BG] to be the right, well-posed, mathematical model to describe one-dimensional pressureless gas with sticky particles (for which a recent reference is [BJL]). In particular, the Eulerian fields $(h, h v)$ defined by

$$
h(t, x)=\partial_{x} u(t, x), \quad h v(t, x)=\partial_{x}(F(u(t, x))
$$

are weak solutions of the pressureless gas equations:

$$
\partial_{t} h+\partial_{x}(h v)=0, \quad \partial_{t}(h v)+\partial_{x}\left(h v^{2}\right)=0 .
$$

Using the same arguments as in section 8 , we claim that a gas of sticky particles exactly corresponds to the vanishing viscosity limit $\varepsilon \rightarrow 0$ of any pressureless viscous model:

$$
\partial_{t} h+\partial_{x}(h v)=0, \quad \partial_{t}(h v)+\partial_{x}\left(h v^{2}\right)=\varepsilon \partial_{x}\left(\mu(h) \partial_{x} v\right),
$$


with positive viscosity $\mu$.

Finally, our analysis provides a new formulation -in Lagrangian coordinates- of a pressureless gas of sticky particles, or, equivalently, of a scalar conservation law with monotonic initial data. Namely:

$$
\partial_{t t} X=-\partial_{t} \partial_{a} \mu, \quad \partial_{a} X \geq 0, \quad \mu \geq 0, \quad \mu \partial_{a} X=0 .
$$

A more precise definition, from which both existence and uniqueness can be easily deduced, is:

Definition 9.1. Let us denote by $\|\cdot\|$ the norm on $L^{2}([0,1])$.

Let $X_{0} \in L^{2}([0,1])$ such that $\partial_{a} X_{0} \geq 0$. We say that

$$
(t, a) \in \mathbf{R}_{+} \times[0,1] \rightarrow X(t, a) \in \mathbf{R}
$$

describes a pressureless gas of sticky particles, with initial value $X_{0}$ and velocity $v_{0}$, if:

i) $X(t, \cdot)$ depends continuously on $t$ with respect to the weak topology of $L^{2}([0,1])$, and $\partial_{a} X \geq 0$ holds true,

ii) $X(0)=X_{0}$,

iii) for all $T \geq 0$ and all smooth functions $Y(t, a)$ such that $\partial_{a} Y \geq 0$,

$$
\|X(T)-Y(T)\|^{2} \leq\left\|X_{0}-Y(0)\right\|^{2}+2 \int_{0}^{T} d t \int_{0}^{1}(X-Y)(t, a)\left(v_{0}(a)-\partial_{t} Y(t, a)\right) d a .
$$

10. Appendix: Proof of Proposition 1.1. To prove Proposition 1.1, we first observe that the two last equations in (3) are compatibility conditions that automatically follow from definition (4). Indeed, given a test function $\phi(x)$, we have, by definition (4),

$$
\int_{\mathbf{R}} \phi(x) h(t, x, y) d x=\int_{0}^{1} \phi(X(t, y, a)) d a .
$$

Thus,

$$
\begin{aligned}
\partial_{t} \int_{\mathbf{R}} \phi(x) h(t, x, y) d x & =\int_{0}^{1} \phi^{\prime}(X(t, y, a)) \partial_{t} X(t, y, a) d a \\
& =\int_{\mathbf{R}} \phi^{\prime}(x) h(t, x, y) v(t, x, y) d x
\end{aligned}
$$

(by definition of $h v$ through (4)) and

$$
\partial_{t} h+\partial_{x}(h v)=0
$$

follows. Similarly, by definition of $h b$ and $h v$ through (4), we have:

$$
\begin{aligned}
& \partial_{t} \int_{\mathbf{R}} \phi(x) h(t, x, y) b(t, x, y) d x-\partial_{y} \int_{\mathbf{R}} \phi(x) h(t, x, y) v(t, x, y) d x \\
& =\int_{0}^{1} \partial_{t}\left(\partial_{y} X(t, y, a) \phi(X(t, y, a))\right) d a-\int_{0}^{1} \partial_{y}\left(\partial_{t} X(t, y, a) \phi(X(t, y, a))\right) d a=0
\end{aligned}
$$

and deduce:

$$
\partial_{t}(h b)-\partial_{y}(h v)=0
$$


Let us now establish that $h, b, v$ satisfy the first equation in (3). First, observe, using (2), that

$$
\int\left(\left(\partial_{y} X\right)^{2},\left(\partial_{t} X\right)^{2}\right) \delta(x-X(t, a, y)) d a=\left(h b^{2}, h v^{2}\right)(t, x, y) .
$$

Thus,

$$
\partial_{t}(h v)=\int \partial_{t t} X \delta(x-X(t, a, y)) d a-\int\left(\partial_{t} X\right)^{2} \delta^{\prime}(x-X(t, a, y)) d a
$$

(by definition (4) of $h v$ )

$$
=\int \partial_{y y} X \delta(x-X(t, a, y)) d a-\int\left(\partial_{t} X\right)^{2} \delta^{\prime}(x-X(t, a, y)) d a
$$

(using equation (1) with $c=1$ )

$$
=\int\left(\left(\partial_{y} X\right)^{2}-\left(\partial_{t} X\right)^{2}\right) \delta^{\prime}(x-X(t, a, y)) d a=\partial_{x}\left(h\left(b^{2}-v^{2}\right)\right) .
$$

The proof of Proposition 1.1 is now complete.

11. Appendix: Derivation of equations (32). Let us use (4) and (2) to express the Eulerian fields $(h, b, v)$ from $(X, U)$. As in section 10, we get from (31), first,

$$
\partial_{t} h+\partial_{x}(h v)=0, \quad \partial_{t}(h b)-\partial_{y}(h v)=0,
$$

and, next,

$$
\begin{aligned}
\partial_{t}(h v)+\partial_{x}\left(h\left(v^{2}-b^{2}\right)\right) & =\int\left(\partial_{t t} X-\partial_{y y} X\right) \delta(x-X(t, a, y)) d a \\
& =\varepsilon \int \partial_{t a}\left(\phi^{\prime}\left(\partial_{a} X\right)\right) \delta(x-X(t, a, y)) d a
\end{aligned}
$$

(because of (31))

$$
\begin{aligned}
& =\varepsilon \int \partial_{a}\left(\phi^{\prime \prime}\left(\partial_{a} X\right) \partial_{t a} X\right) \delta(x-X) d a \\
& =\varepsilon \int \partial_{a}\left(\phi^{\prime \prime}\left(\partial_{a} X\right) \partial_{a}(v(t, X(t, a, y), y)) \delta(x-X) d a\right.
\end{aligned}
$$

(using (2))

$$
\begin{aligned}
& =\varepsilon \int \partial_{a}\left(\phi^{\prime \prime}\left(\partial_{a} X\right)\left(\partial_{x} v\right)(t, X(t, a, y), y) \partial_{a} X\right) \delta(x-X) d a \\
& =\varepsilon \int \partial_{a}\left(\left(\mu(1 / h) \partial_{x} v\right)(t, X, y)\right) \delta(x-X) d a
\end{aligned}
$$

(by definition (33) of $\mu$ and because of (2))

$$
\begin{aligned}
& =\varepsilon \int\left(\partial_{x}\left(\mu(1 / h) \partial_{x} v\right)\right)(t, X, y) \partial_{a} X \delta(x-X) d a \\
& \left.=\varepsilon \partial_{x}\left(\mu(1 / h) \partial_{x} v\right)\right)(t, x, y) \int \partial_{a} X \delta(x-X) d a \\
& \left.=\varepsilon \partial_{x}\left(\mu(1 / h) \partial_{x} v\right)\right)(t, x, y)
\end{aligned}
$$

for $X(t, 0, y)<x<X(t, 1, y)$. Thus (32) holds true, as announced. 
Acknowledgment. This work is partly supported by the European network RTN HYKE and the LRC CEA-Cadarache/UNSA. It originated while the author was visiting the Courant Institute on September 2003 and was continued at the ICES (University of Texas, Austin) on February 2004. The author is very grateful to Luis Caffarelli, Irene Gamba, Nader Masmoudi and Jalal Shatah for their invitation at UT Austin and NYU. He also thank Florent Berthelin, Nader Masmoudi and Bertrand Maury for very stimulating discussions on sticky strings and sticky particles.

\section{REFERENCES}

[BDLL] G. Boillat. C. Dafermos, P. Lax, T.P. Liu, Recent mathematical methods in nonlinear wave propagation, Lecture Notes in Math., 1640, Springer, Berlin, 1996.

[BI] M. Born, L. Infeld, Foundations of the new field theory, Proc. Roy. Soc. London, A 144 (1934), pp. 425-451.

[BJL] F. Bouchut, S. JIN, X. LI, Numerical approximations of pressureless and isothermal gas dynamics, SIAM J. Numer. Anal., 41 (2003), pp. 135-158.

[Br] Y. Brenier, Hydrodynamic structure of the augmented Born-Infeld equations, Archive Rat. Mech. Analysis, 172 (2004), pp. 65-91.

[Br2] Y. BRENIER, Résolution d'équations d'évolution quasilinéaires en dimension $N$ d'espace à l'aide d'équations linéaires en dimension $N+1$, J. Differential Equations, 50 (1983), pp. 375-390.

[Br3] Y. BRENIER, Averaged multivalued solutions for scalar conservation laws, SIAM J. Numer. Anal., 21 (1984), pp. 1013-1037.

[BG] Y. Brenier, E. Grenier, Sticky particles and scalar conservation laws, SIAM J. Numer. Anal., 35 (1998), pp. 2317-2328.

[Bz] H. Brezis, Opérateurs maximaux monotones, et semi-groupes de contractions dans les espaces de Hilbert, North-Holland, Amsterdam, 1973.

[Gib] G. GibBons, Pulse propagation in Born-Infeld theory, the world-volume equivalence principle and the Hagedorn-like equation of state of the Chaplygin gas, http://arxiv.org/pdf/hep-th/0104015, Gravit. Cosmol., 8 (2002), no. 1-2, pp. 2-6.

[Gil] P. Gilman, MHD "shallow water" equations for the solar tachocline, Astrophys. J. Lett., 544,79 (2000).

[GKMP] V. Gorini, A. Kamenshchik, U. Moschella, V. Pasquier, The Chaplygin gas as a model for dark energy, http://arxiv.org/pdf/gr-qc/0403062.

[Ja] J.D. JACKSON, Classical Electrodynamics, section I.3, John Wiley and Sons, second edition, 1975.

[PS] L. Paoli, M. Schatzman, A numerical scheme for impact problems, II. The multidimensional case, SIAM J. Numer. Anal., 40 (2002), pp. 734-768.

[Se] D. Serre, Systems of conservation laws, 1 and 2, ch. 9.6 and 10.1, Cambridge University Press, Cambridge, 2000. 